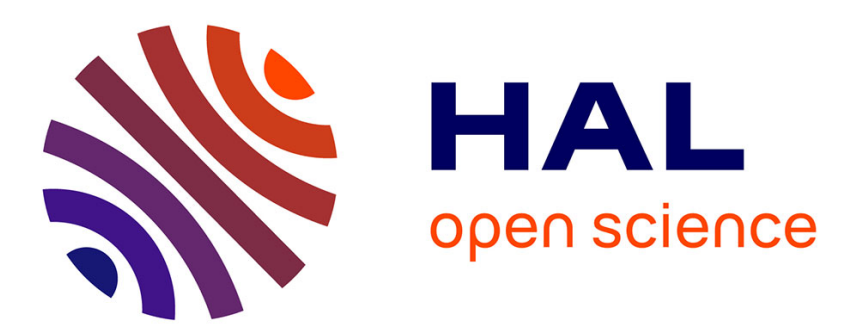

\title{
A State Dependent Sampling for Linear State Feedback
}

Christophe Fiter, Laurentiu Hetel, Wilfrid Perruquetti, Jean-Pierre Richard

\section{To cite this version:}

Christophe Fiter, Laurentiu Hetel, Wilfrid Perruquetti, Jean-Pierre Richard. A State Dependent Sampling for Linear State Feedback. Automatica, 2012, 48 (8), pp.1860-1867. 10.1016/j.automatica.2012.05.063 . hal-00685345

\section{HAL Id: hal-00685345 \\ https://hal.science/hal-00685345}

Submitted on 4 Apr 2012

HAL is a multi-disciplinary open access archive for the deposit and dissemination of scientific research documents, whether they are published or not. The documents may come from teaching and research institutions in France or abroad, or from public or private research centers.
L'archive ouverte pluridisciplinaire HAL, est destinée au dépôt et à la diffusion de documents scientifiques de niveau recherche, publiés ou non, émanant des établissements d'enseignement et de recherche français ou étrangers, des laboratoires publics ou privés. 


\title{
A State Dependent Sampling for Linear State Feedback *
}

\author{
Christophe Fiter ${ }^{\mathrm{a}, *}$ Laurentiu Hetel ${ }^{\mathrm{a}}$ Wilfrid Perruquetti ${ }^{\mathrm{a}, \mathrm{b}}$ Jean-Pierre Richard ${ }^{\mathrm{a}, \mathrm{b}}$ \\ ${ }^{\text {a } L a b o r a t o i r e ~ d ' A u t o m a t i q u e, ~ G e ́ n i e ~ I n f o r m a t i q u e ~ e t ~ S i g n a l ~(C N R S ~ U M R ~ 8219), ~}$ \\ École Centrale de Lille, 59651 Villeneuve d'Ascq, France \\ ${ }^{\mathrm{b}}$ Non-A, INRIA Lille-Nord Europe, France
}

\begin{abstract}
In this work, a new state-dependent sampling control enlarges the sampling intervals of state feedback control. We consider the case of linear time invariant systems and guarantee the exponential stability of the system origin for a chosen decay rate. The approach is based on LMIs obtained thanks to sufficient Lyapunov-Razumikhin stability conditions and follows two steps. In the first step, we compute a Lyapunov-Razumikhin function that guarantees exponential stability for all time-varying sampling intervals up to some given bound. This value can be used as a lower-bound of the state-dependent sampling function. In a second step, an off-line computation provides a mapping from the state-space into the set of sampling intervals: the state is divided into a finite number of regions, and to each of these regions is associated an allowable upper-bound of the sampling intervals that will guarantee the global (exponential or asymptotic) stability of the system. The results are based on sufficient conditions obtained using convex polytopes. Therefore, they involve some conservatism with respect to necessary and sufficient conditions. However, at each of the two steps, an optimization on the sampling upper-bounds is proposed. The approach is illustrated with numerical examples from the literature for which the number of actuations is shown to be reduced with respect to the periodic sampling case.
\end{abstract}

Key words: networked/embedded control systems, state dependent sampling, self-triggered control, Lyapunov-Razumikhin, linear matrix inequality, convex polytope, exponential stability

\section{Introduction}

In the last decades, a large attention has been given to Embedded and Networked Control Systems [20]. From the control theory point of view, they bring up new challenges since they are often required to share a limited number of computational and transmission resources. In practice, this often leads to fluctuations of the sampling interval, which may have a destabilizing effect if it is not properly taken into account. Several studies about this robustness aspect have been made (see [5],

\footnotetext{
* This work was supported by Ministry of Higher Education and Research, Nord-Pas de Calais, and FEDER through the 'CPER 2007-2013', by the European Community's Seventh Framework Programme (FP7/2007-2013) under grant agreement $n^{\circ}$ 257462: HYCON2 Network of Excellence "HighlyComplex and Networked Control Systems", and by the INTERREG IV A 2 Mers Seas Zeen Cross-border Cooperation Program 2007-2013 under SYSIASS 6-20 project.

* Corresponding author. Tel.: +33 (0)3 20676032

Email address:

christophe.fiter@centraliens-lille.org (Christophe Fiter).
}

[17], [7], [6], [11], and [4]). Recently, intensive research has also been conducted to adapt dynamically the sampling in order to reduce the processor and/or network loads while ensuring the desired control performances. There are two main approaches in the literature:

Event-triggered control [18], [8], [13]: intelligent sensors send information to the controller when special events occur (e.g. crossing a frontier of the state space). This requires a dedicated hardware.

Self-triggered control [15], [1], [19]: emulates eventtriggered control without dedicated hardware. At each sampling instant, one computes a lower-bound of the next admissible sampling interval. [15] considers the case of perturbed linear systems and ensures their exponential input-to-state stability using a method based on a discretization of the event generator. [1] studies the case of input-to-state stability for unperturbed homogeneous or polynomial systems using innovative scaling laws on the system's homogeneous rays. [19] treats the $\mathcal{L}_{2}$-stability of delayed perturbed systems. In these works, the computations for the sampling law are made online. Also, it is still an open problem to compute the Lyapunov function while optimizing the sampling intervals. 
In this paper, the design of the sampling rule is made offline, once for all. This enables to trade online computations for offline computations, and thus makes it possible to reduce the number of computations made during the real-time control of the system. We provide an LMI method based on sufficient Lyapunov-Razumikhin stability conditions which enables

- to compute the adequate Lyapunov-Razumikhin function (LRF), in order to enlarge the lower-bound of the sampling function,

- to design a lower-bound approximation of the associated maximal sampling function.

The Lyapunov-Razumikhin type of the proposed stability conditions is suggested by the delayed nature of the system, since it uses a Zero-Order-Hold control [6]. Its design is based on convexification arguments [10], the conservatism of which may be tuned according to the numerical complexity. In this work, we will consider only simple quadratic LRFs.

The paper is organized as follows. First, we state the problem in Section 2. Then, Sections 3 and 4 describe the proposed method and the guaranteed performances. Finally, some simulation results are shown in Section 5 before concluding in Section 6. All the proofs are given in the Appendix, together with technical descriptions.

Notations: We denote $\mathbb{R}_{+}=\{\lambda \in \mathbb{R}, \lambda \geq 0\}$, and $\mathbb{R}^{*}=\{\lambda \in \mathbb{R}, \lambda \neq 0\} . \mathcal{M}_{n}(\mathbb{R})$ is the set of $n \times n$ matrices, the notation $P \succ 0$ (resp. $P \succeq 0$ ) for a symmetric matrix $P \in \mathcal{M}_{n}(\mathbb{R})$ means that $P$ is positive definite (resp. positive semidefinite), and the largest (resp. lowest) eigenvalue of $M \in \mathcal{M}_{n}(\mathbb{R})$ is denoted $\lambda_{\max }(M)$ (resp. $\left.\lambda_{\min }(M)\right)$. We also denote by $\lfloor x\rfloor$ the largest integer not greater than $x: x-1<\lfloor x\rfloor \leq x$. $\|$.$\| stands for$ the Euclidean norm.

\section{Problem statement and preliminaries}

Consider the linear time invariant (LTI) system

$$
\begin{aligned}
& \dot{x}(t)=A x(t)+B u(t), \forall t \in \mathbb{R}_{+} \\
& x(t)=x_{0}, \forall t \leq 0,
\end{aligned}
$$

where $x: \mathbb{R} \rightarrow \mathbb{R}^{n}$ and $u: \mathbb{R}_{+} \rightarrow \mathbb{R}^{n_{u}}$ represent the system state and the control function, and the matrices $A$ and $B$ are constant and of appropriate dimensions. The control is a piecewise-constant state feedback

$$
u(t)=-K x\left(t_{k}\right), \forall t \in\left[t_{k}, t_{k+1}\right),
$$

where $K$ is fixed and such that $A-B K$ is Hurwitz, and where $0=t_{0}<t_{1}<\cdots<t_{k}<\cdots$ are the sampling instants satisfying $\lim _{k \rightarrow \infty} t_{k}=\infty$ and defined by

$$
t_{k+1}=t_{k}+\tau\left(x\left(t_{k}\right)\right), \forall k \in \mathbb{N}
$$

with a state dependent sampling function $\tau: \mathbb{R}^{n} \rightarrow \mathbb{R}_{+}$. To ensure the well-posedness during the whole work, the function is assumed to be lower-bounded by some scalar $\delta>0$. We denote by $\mathcal{S}$ the closed-loop system $\{(1),(2)$, (3) $\}$. For a given sampling function $\tau$, the solution of $\mathcal{S}$ with initial value $x_{0}$ is denoted by $x(t)=\varphi_{\tau}\left(t, x_{0}\right)$.

In this work, our main objective is to provide a way to enlarge as much as possible the state dependent sampling function $\tau$ in (3) while ensuring the exponential stability of the system's origin for a chosen decay rate $\beta$, also called $\beta$-stability (i.e. such that there exists a scalar $\gamma$ for which all trajectories satisfy $\|x(t)\| \leq \gamma \mathrm{e}^{-\beta t}\left\|x_{0}\right\|$ for any initial condition $x_{0}$ ).

In order to check out the system's $\beta$-stability, we use an LRF approach [12].

Proposition 1 Given scalars $\alpha>1, \bar{\sigma}>0$, and $0<$ $\beta \leq \frac{\ln (\alpha)}{2 \bar{\sigma}}$, if there exist a quadratic function $V(x)=$ $x^{T} P x, P=P^{T} \succ 0 \in \mathcal{M}_{n}(\mathbb{R})$, and a function $\tau: \mathbb{R}^{n} \rightarrow$ $\mathbb{R}_{+}, 0<\delta \leq \tau(x) \leq \bar{\sigma}$, such that

(C1): "for all $x \in \mathbb{R}^{n}$, for all $\sigma \in[0, \tau(x)], \dot{V}\left(\varphi_{\tau}(\sigma, x)\right)+$ $2 \beta V\left(\varphi_{\tau}(\sigma, x)\right) \leq 0$ whenever $\alpha V\left(\varphi_{\tau}(\sigma, x)\right) \geq V(x)$ ", then the origin of $\mathcal{S}$ is globally $\beta$-stable.

Note that if $\beta=0$ and the inequality $\dot{V}\left(\varphi_{\tau}(\sigma, x)\right) \leq 0$ in $(\mathrm{C} 1)$ is reinforced to be strict, then the classical LRF [12] theory ensures the system's asymptotic stability.

It is also important to note that all the stability properties in this paper can be extended to state-dependent time-varying samplings $t_{k+1}=t_{k}+\tilde{\tau}\left(t_{k}, x\left(t_{k}\right)\right), \forall k \in \mathbb{N}$, with a time-varying sampling function $\tilde{\tau}: \mathbb{R}_{+} \times \mathbb{R}^{n} \rightarrow$ $\mathbb{R}_{+}$. The closed-loop system $\{(1),(2)\}$ with such a sampling law is denoted $\tilde{\mathcal{S}}$.

Proposition 2 If there exist functions $V$ and $\tau$ satisfying condition (C1) in Proposition 1, then the origin of $\tilde{\mathcal{S}}$ is globally $\beta$-stable for any time-varying sampling function $\tilde{\tau}: \mathbb{R}_{+} \times \mathbb{R}^{n} \rightarrow \mathbb{R}_{+}$satisfying $0<\delta \leq \tilde{\tau}(t, x) \leq \tau(x)$ for all $t \in \mathbb{R}_{+}$and for all $x \in \mathbb{R}^{n}$.

These two propositions are proven in the Appendix. Throughout this work, we will focus on solving two main problems. The first problem concerns the design of the sampling function and is formulated as:

Problem 1: For a given system $\{(1),(2)\}$ and LRF $V$, we denote $\tau_{\text {opt }}^{V}(x)$ the maximal sampling function such that $(\mathrm{C} 1)$ holds: $\tau_{\text {opt }}^{V}(x)=\max \tau(x)$.

Find a lower-bound approximation of this optimal function, $\tau_{\text {sub }}^{V}(x) \leq \tau_{\text {opt }}^{V}(x)$, as large as possible.

In that formulation, the LRF is supposed to be given, which makes us wonder if there is a clever way to choose 
it. Since the objective is to sample as few times as possible, one will also want to make sure the minimal sampling interval is as large as possible by solving the following problem:

Problem 2: For a given system $\{(1),(2)\}$, we denote $\tau_{\text {opt }}^{*}$ the maximal lower-bound of the sampling functions satisfying $(\mathrm{C} 1): \tau_{\text {opt }}^{*}=\operatorname{maxinf}_{x \in \mathbb{R}^{n}} \tau(x)$.

Find an LRF $V$ ensuring (C1) for a sampling function with a lower-bound $\tau_{\text {sub }}^{*} \leq \tau_{\text {opt }}^{*}$ as large as possible.

In order to provide tractable stability conditions from Proposition 1, we first introduce the following Lemma:

Lemma 3 Given scalars $\alpha>1, \bar{\sigma}>0$, and $0<\beta \leq$ $\frac{\ln (\alpha)}{2 \bar{\sigma}}$, if there exist a matrix $P=P^{T} \succ 0 \in \mathcal{M}_{n}(\mathbb{R})$, a scalar $\varepsilon \geq 0$, and a function $\tau: \mathbb{R}^{n} \rightarrow \mathbb{R}_{+}, 0<\delta \leq$ $\tau(x) \leq \bar{\sigma}$, such that for all $x \in \mathbb{R}^{n}$, for all $\sigma \in[0, \tau(x)]$,

$$
x^{T} \Phi(\sigma) x \leq 0,
$$

with

$$
\begin{gathered}
\Phi(\sigma)=\left(\begin{array}{c}
\Lambda(\sigma) \\
I
\end{array}\right)^{T} \Omega\left(\begin{array}{c}
\Lambda(\sigma) \\
I
\end{array}\right), \\
\Omega=\left(\begin{array}{rr}
A^{T} P+P A+\varepsilon \alpha P+2 \beta P-P B K \\
-K^{T} B^{T} P
\end{array}\right),
\end{gathered}
$$

and

$$
\Lambda(\sigma)=I+\int_{0}^{\sigma} e^{s A} d s(A-B K),
$$

then the origin of $\mathcal{S}$ is globally $\beta$-stable.

Remark 1: At the sampling instants, $\Phi(0)=(A-$ $B K)^{T} P+P(A-B K)+\varepsilon(\alpha-1) P+2 \beta P$. If the matrix $P$ is such that $(A-B K)^{T} P+P(A-B K) \prec 0$ (there exists such $P$ since $A-B K$ is Hurwitz), we can find $\varepsilon$ and $\beta$ as small as needed such that $\Phi(0) \prec 0$. Since the function that associates the eigenvalues of $\Phi(\sigma)$ with each time $\sigma$ is continuous on $[0, \tau(x)]$, there exists a scalar $\delta>0$ such that $\Phi(\sigma) \preceq 0$ for all $\sigma \in[0, \delta]$. Therefore, with these parameters, there always exist sampling functions $\tau$ that satisfy Lemma 3 conditions, and which are lowerbounded by some scalar $\delta>0$, hence avoiding any Zeno phenomenon issue.

Remark 2: The conditions of Lemma 3 are the same for a state $x \neq 0$ and for $\lambda x, \lambda \in \mathbb{R}^{*}$. Therefore, it is sufficient to work with homogeneous state dependent sampling functions of degree 0 (i.e. satisfying $\tau(\lambda x)=$ $\tau(x)$ for all $x \in \mathbb{R}^{n}, \lambda \in \mathbb{R}^{*}$ ) and to check Lemma 3 stability conditions on the unit $n$-sphere.

Lemma 3 gives some preliminary stability conditions for a state feedback control system with a state dependent sampling. However, one can see that there is an infinite number of inequalities to check because of both temporal and spatial dependencies in the stability conditions.

\section{Main results}

To derive a finite number of stability conditions from Lemma 3, a two-step tractable methodology is proposed:

- Conic covering of the state space: The space is covered by a set of $q$ conic regions

$$
\mathcal{R}_{s}=\left\{x \in \mathbb{R}^{n}, x^{T} Q_{s} x \geq 0\right\}, Q_{s}=Q_{s}^{T} \in \mathcal{M}_{n}(\mathbb{R}) .
$$

Two possible constructions of these conic regions are presented in Appendix 7.2. To each of these regions $\mathcal{R}_{s}$ corresponds a sampling interval $\tau_{s}>0$ that we want to maximize. The state dependent sampling function can

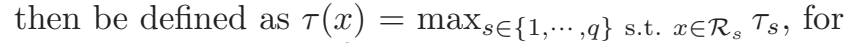
all $x \in \mathbb{R}^{n}$. The use of a conic covering is motivated by the homogeneity brought up in Remark 2.

- Convex embedding according to time: Let $s \in$ $\{1, \cdots, q\}$. The matrix function $\Phi(\sigma)$ is continuous on the compact set $\left[0, \tau_{s}\right]$. Therefore, it is possible to build a convex polytope defined by a finite set of vertices $\Phi_{\kappa, s}$, $\kappa \in \mathcal{K}_{s}$ (a finite set of indexes), such that for any $x \in \mathcal{R}_{s}$

$$
\begin{aligned}
& \left(x^{T} \Phi_{\kappa, s} x \leq 0, \forall \kappa \in \mathcal{K}_{s}\right) \\
& \Rightarrow\left(x^{T} \Phi(\sigma) x \leq 0, \forall \sigma \in\left[0, \tau_{s}\right]\right) .
\end{aligned}
$$

The form of the matrix function $\Phi$ given by (5) enables to build these vertices as linearly dependent on $P$, and dependent on the parameters $\alpha, \varepsilon, \beta$, and $\bar{\sigma}$. One possible construction of a convex polytope satisfying (9) is provided in Appendix 7.3, Lemma 6 (equations (14) to (19)). This construction is based on the results from [10], which provide tools to build convex hulls around exponential matrix functions using Taylor polynomials.

Both of these two steps introduce some conservatism in Lemma 3 stability conditions. The conservatism introduced by the convex embedding is discussed in [9], while the one coming from the conic covering is discussed in Appendix 7.2.

Using these steps, we derive the following Theorem.

Theorem 4 Let a matrix $P=P^{T} \succ 0 \in \mathcal{M}_{n}(\mathbb{R})$, and scalars $\varepsilon \geq 0, \alpha>1, \bar{\sigma}>0$, and $0<\beta \leq \frac{\ln (\alpha)}{2 \bar{\sigma}}$ be given. Consider the conic regions (8), sampling intervals $\tau_{1}, \cdots, \tau_{q}$ satisfying $0<\tau_{s} \leq \bar{\sigma}$, and matrices $\Phi_{\kappa, s}$ satisfying (9), for all $s \in\{1, \cdots, q\}, \kappa \in \mathcal{K}_{s}$. The sampling function $\tau: \mathbb{R}^{n} \rightarrow \mathbb{R}_{+}$is defined as $\tau(x)=\tau_{s}$ for all $x \in \mathcal{R}_{s}$ and $s \in\{1, \cdots, q\}$.

If there exist scalars $\varepsilon_{\kappa, s} \geq 0$ such that the LMIs

$$
\Phi_{\kappa, s}+\varepsilon_{\kappa, s} Q_{s} \preceq 0
$$

are satisfied for all $s \in\{1, \cdots, q\}$ and $\kappa \in \mathcal{K}_{s}$, then the origin of $\mathcal{S}$ is globally $\beta$-stable. 
Theorem 4 provides sufficient conditions for Lemma 3, which enables to compute a lower-bound approximation of the optimal sampling function $\tau_{\mathrm{opt}}^{V}$ (i.e. a solution to Problem 1).

Remark 3: From Theorem 4 and Proposition 2, similar results can be obtained for any time-varying sampling function $\tilde{\tau}: \mathbb{R}_{+} \times \mathbb{R}^{n} \rightarrow \mathbb{R}_{+}$satisfying $0<\delta \leq \tilde{\tau}(t, x) \leq$ $\tau(x)$ for all $t \in \mathbb{R}_{+}$and for all $x \in \mathbb{R}^{n}$.

Corollary 5 Consider a covering of the state space composed of one single region $\mathcal{R}=\mathbb{R}^{n}$. Consider $\varepsilon \geq 0$ a tuning parameter. Let scalars $\alpha>1, \bar{\sigma}>0$, and $0<\beta \leq \frac{\ln (\alpha)}{2 \bar{\sigma}}$, and matrices $\Phi_{\kappa}$ satisfying (9), with $\kappa \in \mathcal{K}$ (the indexes $s$ denoting the regions in (9) are dropped since we consider only one region: $\left.\mathcal{R}=\mathbb{R}^{n}\right)$. Let us assume that the sampling function $\tau: \mathbb{R}^{n} \rightarrow \mathbb{R}_{+}$ satisfies $\tau(x)=\tau^{*}$ for all $x \in \mathbb{R}^{n}$, for a given scalar $0<\tau^{*} \leq \bar{\sigma}$.

If there exists a matrix $P=P^{T} \succ 0 \in \mathcal{M}_{n}(\mathbb{R})$ such that the LMIs $\Phi_{\kappa} \preceq 0$ are satisfied for all $\kappa \in \mathcal{K}$, then the origin of system (1) is globally $\beta$-stable regarding the control (2) for any time-varying sampling bounded by $\tau^{*}$.

Remark 4: For a given value of $\varepsilon$, one can compute the maximal $\tau^{*}$ (denoted $\tau_{\varepsilon}^{*}$ ) for which the stability conditions from Corollary 5 are satisfied, by using a line search algorithm on the variable $\tau^{*}$ and LMI solvers. Another line search algorithm is then used on the variable $\varepsilon$ so as to compute an estimation of the largest upper-bound for time-varying samplings: $\tau_{\mathrm{sub}}^{*}=\sup _{\varepsilon \geq 0} \tau_{\varepsilon}^{*}$.

Remark 5: The state independent Corollary 5 can be used to compute: an upper-bound estimation $\tau_{\text {sub }}^{*}$ for time-varying samplings as in the framework of robust control techniques (i.e. guaranteeing $\beta$-stability for any time-varying sampling bounded by $\tau_{\text {sub }}^{*}$ ), which is also a lower-bound estimation of $\tau_{\text {opt }}^{*}$ (i.e. a solution to Problem 2); the LRF $V(x)=x^{T} P x$ used for the state dependent sampling design (in Theorem 4).

\section{General algorithm}

Theorem 4 and Corollary 5 may be used to solve Problems 1 and 2 respectively. While Corollary 5 gives a way to compute the LRF parameters $P$ and $\varepsilon$ maximizing an estimation of the lower-bound $\tau^{*}$ of the sampling function $\tau$ under the stability conditions of Proposition 1, Theorem 4 gives a way to approximate the sampling function $\tau_{\mathrm{opt}}^{V}$ on state regions, for given $P$ and $\varepsilon$. A method to apply the proposed technique is the following:

First, use Corollary 5 and the polytopic description (14) with $\nu=0$. Then, the research for $P$ is an LMI problem, and we may optimize the search of a lower-bound estimate $\hat{\tau}_{\text {sub }}^{*}$ of $\tau_{\text {opt }}^{*}$ as well as its associated $\varepsilon$ using the technique proposed in Remark 4.
Next, we compute the value $\nu$ assigned to the obtained $P$ and $\varepsilon$, and we evaluate the matrix inequalities $\Phi_{\kappa} \preceq 0$ in Corollary 5 so as to obtain the value $\tau_{\text {sub }}^{*} \leq \hat{\tau}_{\text {sub }}^{*}$ which satisfies the stability conditions.

Finally, the LMI conditions from Theorem 4 are used with the computed values of $P, \varepsilon$ and $\nu$ to approximate the maximal state dependent sampling function $\tau_{\text {opt }}^{V}$ (i.e. $\tau_{\text {sub }}^{V}(x)=\max \tau_{s}, \forall x \in \mathcal{R}_{s}, s \in\{1, \cdots, q\}$, such that the LMIs (10) hold). Note that it is possible to solve the LMIs to maximize the sampling times $\tau_{s}$ on each region separately.

Remark 6: This algorithm provides a practical method to build a lower-bound approximation $\tau_{\text {sub }}^{V}$ of the optimal sampling function $\tau_{\mathrm{opt}}^{V}$. As most of the numerical methods, there is no a priori evaluation of the gap between the obtained function and the optimal function. However, the benefits of this technique are shown for some benchmarks from the literature in Section 5.

\section{Numerical examples}

\subsection{Example 1}

Consider the following system from [11]:

$$
\begin{aligned}
& \dot{x}(t)=\left(\begin{array}{cc}
-0.5 & 0 \\
0 & 3.5
\end{array}\right) x(t)-\left(\begin{array}{l}
1 \\
1
\end{array}\right) K x\left(t_{k}\right), \\
& K=\left(\begin{array}{ll}
-1.02 & 5.62
\end{array}\right) .
\end{aligned}
$$

After setting the polynomial approximation degree term $N=5$, the number of polytopic subdivisions $l=100$, and the number of equal conic regions $q=100$ (isotropic covering on the unit sphere $x=\mathrm{e}^{\mathrm{i} \theta}, \theta \in[-\pi, \pi]$, see Appendix 7.2.1), we can obtain a mapping of the state space that gives the maximal allowable sampling interval for each state for a given decay rate $\beta>0$ thanks to Corollary 5 and Theorem 4 . For each $\beta$, after fixing $\bar{\sigma}$, we set the LRF performance parameter $\alpha>1$ (see Proposition 1) as small as possible and such that $\beta \leq \frac{\ln (\alpha)}{2 \bar{\sigma}}$. The state dependent sampling functions obtained offline and ensuring the $\beta$-stability of the system for different decay rates $\beta$ are presented in Figure 1.

For a constant sampling greater than $T_{\max }=0.469 \mathrm{~s}$ the discrete-time dynamic matrix is not Schur anymore, so the system becomes unstable. However, with the proposed technique, we can go beyond the limit $T_{\max }$ for some regions of the state space (up to $1 s$ for $\beta=0$ ).

Figure 2 (resp. Figure 3 ) shows simulation results with $\beta=0$ (resp. $\beta=0.05$ ) and a random initial state. It first shows the sampling intervals (blue/piecewise constant curve), with the lower-bound of the offline computed state dependent sampling function (red/lower 


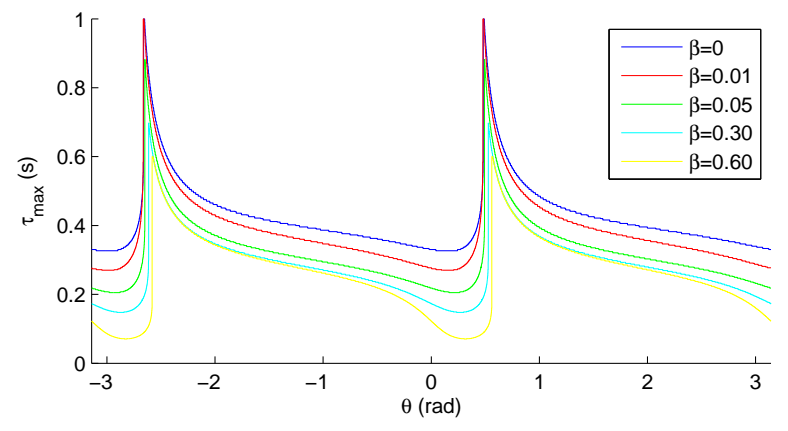

Fig. 1. Example 1: State-angle dependent sampling function $\tau$ for different decay rates $\beta$ (from top to bottom, $\beta=0,0.01,0.05,0.30,0.60)$

horizontal line), and the limit $T_{\max }$ of the periodic case (green/upper horizontal line), before showing the LRF evolution. The sampling times are represented by the red dots on each graph. Note that the evolution of the LRF illustrates the conservatism of the (sufficient) stability conditions from Theorem 4 . For $\beta=0$, for instance (see Figure 2), the triggering condition from Proposition 1 should be $V(x(t))=\frac{V\left(x\left(t_{k}\right)\right)}{\alpha} \simeq V\left(x\left(t_{k}\right)\right)$, when $\dot{V}(x(t))>0$ ( $\alpha$ was set to 1.001). Thus, the gap between $V\left(x\left(t_{k}\right)\right)$ and $V(x(t))$ at the triggering instants in the simulation represents the conservatism of the method.
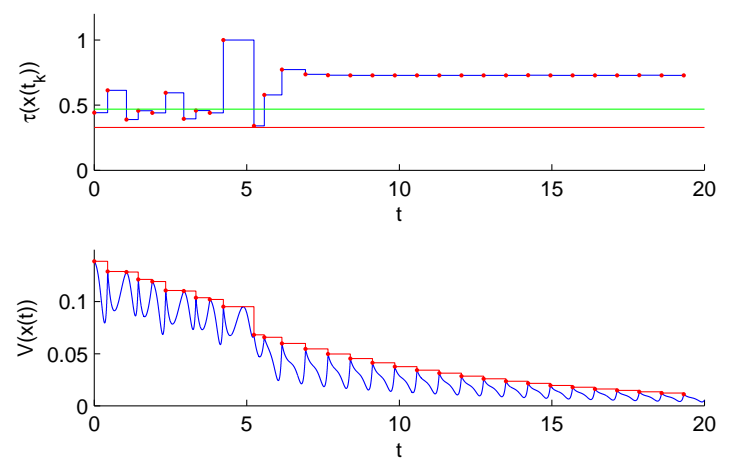

Fig. 2. Example 1: Inter-execution times $\tau\left(x\left(t_{k}\right)\right)$ and LRF $V(x)=x^{T} P x$ for a decay rate $\beta=0$.

In Figure $2(\beta=0)$, one can see that the number of actuations over the $20 \mathrm{~s}$ time interval is 31 instead of 43 with $T_{\max }$. For any (tested) initial condition in the simulation, the average sampling time converges to $T_{\text {average }} \simeq$ $0.726 s \simeq 155 \% T_{\max }$.

For a given decay-rate $\beta>0$, the maximal constant sampling ensuring $\beta$-stability is given by $T_{\max }^{\beta}=$ $\operatorname{argmax}\left\{T>0,-\frac{\ln \left(\left|\lambda_{\max }\right|\right)}{T} \geq \beta\right\}<T_{\max }$, where $\lambda_{\max }$ is the eigenvalue of $A_{d}(T)$ with greatest modulus. In the simulation of Figure $3(\beta=0.05)$, we can observe that $T_{\text {average over 20s }}^{\beta=0.05}=0.486 s>T_{\max }=0.469 \mathrm{~s}>T_{\max }^{\beta=0.05}=$ $0.457 \mathrm{~s}$.

This means that it is possible to sample less in average
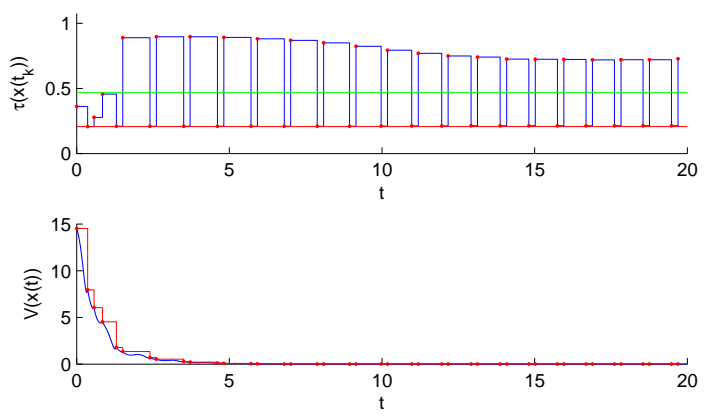

Fig. 3. Example 1: Inter-execution times $\tau\left(x\left(t_{k}\right)\right)$ and LRF $V(x)=x^{T} P x$ for a decay rate $\beta=0.05$.

than with the maximal periodic sampling $T_{\max }$ while still ensuring asymptotic or exponential stability. Although we can not guarantee that this will always be the case, the state-dependent sampling presents some advantages compared to periodic sampling:

- It ensures some convergence performance ( $\beta$-stability for a given decay-rate $\beta$, or asymptotic stability if $\beta=0$ ), whereas constant sampling with $T_{\max }$ only ensures marginal stability and doesn't give any hint about the inter-sampling state behaviour.

- It guarantees robustness regarding possible fluctuations of the sampling period, which is inherent to practical applications (due to scheduling issues for example). The state-dependent sampling approach ensures the system's $\beta$-stability for any time-varying sampling period satisfying $0<\delta \leq \tilde{\tau}(t, x) \leq \tau(x)$, for all $t \in \mathbb{R}_{+}$ and for all $x \in \mathbb{R}^{n}$ (Remark 3$)$.

Note that in many numerical examples, the lower-bound $\tau_{\text {sub }}^{*}$ of the sampling function is usually not far from the value of $T_{\max }$. In the worst case scenario, we can take a constant sampling interval equal to $\tau_{\text {sub }}^{*}$. Also, since Remark 3 ensures asymptotic stability for any time-varying sampling bounded by the designed function $\tau$ with $\beta=0$ (i.e. any time-varying sampling with values under the blue curve in Figure 1), it is also interesting to compare the lower-bound $\tau_{\text {sub }}^{*}=0.329$ (computed using Corollary 5 ) of the designed state dependent sampling function with the maximum upper-bounds obtained in recent papers about (state independent) time-varying sampling: $\tau_{\text {sub }}^{*}=0.165$ [16], 0.198 [17], 0.204 [7], or 0.259 [5].

\subsection{Example 2}

Consider the Batch Reactor system from [14]:

$\dot{x}(t)=\left(\begin{array}{cccc}1.38 & -0.20 & 6.71 & -5.67 \\ -0.58 & -4.29 & 0 & 0.67 \\ 1.06 & 4.27 & -6.65 & 5.89 \\ 0.04 & 4.27 & 1.34 & -2.10\end{array}\right) x(t)+\left(\begin{array}{cc}0 & 0 \\ 5.67 & 0 \\ 1.13 & -3.14 \\ 1.13 & 0\end{array}\right) u(t)$,
$K=\left(\begin{array}{cccc}-0.1006 & 0.2469 & 0.0952 & 0.2447 \\ -1.4099 & 0.1966 & -0.0139 & -0.0823\end{array}\right)$ 


\section{Appendix}

We use the same parameters $N=5$ and $l=100$ as in the previous example, along with $\bar{\sigma}=1 s$ and $q=30$ conic regions built using the method proposed in the Appendix 7.2.2, and design the mapping of the state space for $\beta=0$. This state space mapping (in dimension 4) provides a precise knowledge of the sampling function $\tau$ (which varies from $\tau_{\text {sub }}^{*}=0.4409$ to $0.9883 \leq \bar{\sigma}$ ). In comparison, the value of the maximal allowable constant sampling $T_{\max }$ is $0.5534 \mathrm{~s}$. Using this mapping, we obtain the simulations shown in Figure 4.
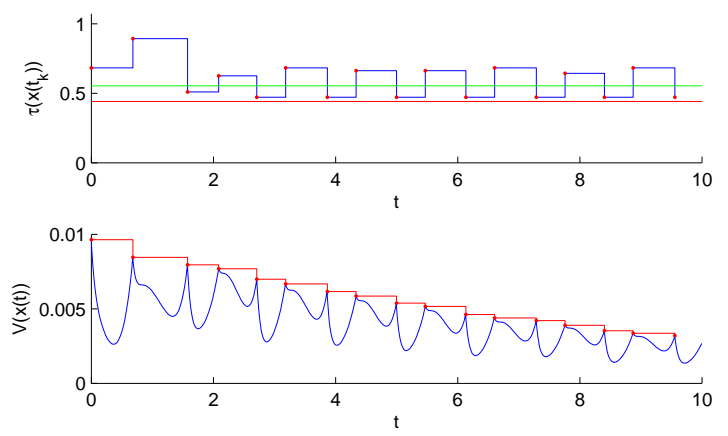

Fig. 4. Example 2: Inter-execution times $\tau\left(x\left(t_{k}\right)\right)$ and LRF $V(x)=x^{T} P x$ for a decay rate $\beta=0$.

The number of actuations over the first $10 \mathrm{~s}$ time interval (see Figure 4) is 17, which can be compared to the number of updates presented in [14] (32 in the best presented case), and the obtained average sampling time is $T_{\text {average }}=0.5898>T_{\max }$.

This example can also be treated via the isotropic conic covering presented in Appendix 7.2.1. With 8000 conic regions, one obtains 21 updates over the first 10s.

\section{Conclusion}

We have introduced an LRF based design for a state dependent sampling function $\tau$ ensuring the exponential stability with a given decay-rate for linear state feedback systems. The proposed method can be seen both as a self-triggered control and as a new time-varying sampling analysis leading to a state dependent sampling design. A lower-bound estimation of the maximal sampling function is proposed. The method presents several advantages.

- It makes it possible to maximize the lower-bound $\tau^{*}$ of the proposed function.

- It provides the associated LRF parameters.

- The real-time implementation takes advantage of an offline designed mapping of the next sampling interval with respect to the past sampled state value.

Extensions to perturbed, delayed, and nonlinear systems, as well as other types of Lyapunov functions to reduce the conservatism, are currently being studied.

\subsection{Proofs}

Proof of Propositions 1 and 2: Let $\alpha>1, \bar{\sigma}>0$ and $\beta>0$ be given. If there exist a quadratic function $V(x)=x^{T} P x, P=P^{T} \succ 0 \in \mathcal{M}_{n}(\mathbb{R})$ and a function $\tau: \mathbb{R}^{n} \rightarrow \mathbb{R}_{+}, 0<\delta \leq \tau(x) \leq \bar{\sigma}$, satisfying the conditions of Proposition 1, then the usual LRF theory [12] adapted to sampled data systems ensures the asymptotic stability of the system origin for both Propositions 1 and 2. Let us take such parameters satisfying (C1).

Let us then consider a time-varying sampling function $\tilde{\tau}: \mathbb{R}_{+} \times \mathbb{R}^{n} \rightarrow \mathbb{R}_{+}$defining sampling instant sequences by the law $t_{k+1}=t_{k}+\tilde{\tau}\left(t_{k}, x\left(t_{k}\right)\right), k \in \mathbb{N}$ and satisfying $0<\delta \leq \tilde{\tau}(t, x) \leq \tau(x)$ for all $t \in \mathbb{R}_{+}$and $x \in \mathbb{R}^{n}$. During a sampling interval $[0, \tilde{\tau}(0, x))$ with initial state $x$, two cases may occur. The first case is that during that time interval, $V\left(\varphi_{\tau}(\sigma, x)\right)$ never goes below $\frac{V(x)}{\alpha}$. Then, the differential inequality $\dot{V}\left(\varphi_{\tau}(\sigma, x)\right)+2 \beta V\left(\varphi_{\tau}(\sigma, x)\right) \leq 0$ is satisfied for all $\sigma \in[0, \tilde{\tau}(0, x))$ according to $(\mathrm{C} 1)$ and therefore $V\left(\varphi_{\tau}(\tilde{\tau}(0, x), x)\right) \leq \mathrm{e}^{-2 \beta \tilde{\tau}(0, x)} V(x)$. In the other case, $V\left(\varphi_{\tau}(\sigma, x)\right)$ manages to go below $\frac{V(x)}{\alpha}$ during that time interval. According to $(\mathrm{C} 1), \dot{V}\left(\varphi_{\tau}(\sigma, x)\right) \leq 0$ over the set $\Upsilon_{x}=\left\{y \in \mathbb{R}^{n}, V(y) \geq \frac{V(x)}{\alpha}\right\}$, and one can show as in the framework of [2] that the set $\bar{\Upsilon}_{x}=\left\{y \in \mathbb{R}^{n}, V(y) \leq \frac{V(x)}{\alpha}\right\}$ is positive invariant. Therefore, if $V\left(\varphi_{\tau}(\sigma, x)\right)$ goes below $\frac{V(x)}{\alpha}$, one will have $V\left(\varphi_{\tau}(\tilde{\tau}(0, x), x)\right) \leq \frac{V(x)}{\alpha}$. Moreover, if $\beta$ satisfies $\beta \leq \frac{\ln (\alpha)}{2 \bar{\sigma}}$, then we get $V\left(\varphi_{\tau}(\tilde{\tau}(0, x), x)\right) \leq \mathrm{e}^{-2 \beta \bar{\sigma}} V(x) \leq$ $\mathrm{e}^{-2 \beta \tilde{\tau}(0, x)} V(x)$.

Therefore, for any initial state $x_{0}$, for any $t \in \mathbb{R}_{+}$, $t \in\left[t_{k}, t_{k+1}\right)$ for some $k \in \mathbb{N}$, one has $V(x(t)) \leq$ $V\left(x\left(t_{k}\right)\right) \leq \mathrm{e}^{-2 \beta \sum_{i=0}^{k-1} \tilde{\tau}\left(t_{i}, x\left(t_{i}\right)\right)} V\left(x_{0}\right)=\mathrm{e}^{-2 \beta t_{k}} V\left(x_{0}\right) \leq$ $\mathrm{e}^{-2 \beta(t-\bar{\sigma})} \bar{V}\left(x_{0}\right)$. As a consequence, one can show that $\|x(t)\| \leq\left(\sqrt{\frac{\lambda_{\max }(P)}{\lambda_{\min }(P)}} \mathrm{e}^{\beta \bar{\sigma}}\right) \mathrm{e}^{-\beta t}\left\|x_{0}\right\|$, which proves the $\beta$-stability of both Propositions 1 (with $\tilde{\tau}(t, x)=\tau(x)$ for all $t \in \mathbb{R}_{+}$and $\left.x \in \mathbb{R}^{n}\right)$ and 2 .

Proof of Lemma 3: Let us take a quadratic function $V(x)=x^{T} P x, P=P^{T} \succ 0 \in \mathcal{M}_{n}(\mathbb{R})$, scalars $\alpha>1$, $\bar{\sigma}>0$, and $0<\beta \leq \frac{\ln (\alpha)}{2 \bar{\sigma}}$, and a function $\tau: \mathbb{R}^{n} \rightarrow \mathbb{R}_{+}$ upper-bounded by $\bar{\sigma}$, and let us rewrite the propositions used in the stability condition of Proposition 1.

Rewrite $\alpha V\left(\varphi_{\tau}(\sigma, x)\right) \geq V(x)$ as

$\left(\begin{array}{c}\varphi_{\tau}(\sigma, x) \\ x\end{array}\right)^{T}\left(\begin{array}{cc}-\alpha P & 0 \\ 0 & P\end{array}\right)\left(\begin{array}{c}\varphi_{\tau}(\sigma, x) \\ x\end{array}\right) \leq 0$,

and $\dot{V}\left(\varphi_{\tau}(\sigma, x)\right)+2 \beta V\left(\varphi_{\tau}(\sigma, x)\right) \leq 0$ as

$\left(\begin{array}{c}\varphi_{\tau}(\sigma, x) \\ x\end{array}\right)^{T}\left(\begin{array}{cc}A^{T} P+P A+2 \beta P & -P B K \\ -K^{T} B^{T} P & 0\end{array}\right)\left(\begin{array}{c}\varphi_{\tau}(\sigma, x) \\ x\end{array}\right) \leq 0$.

Using the lossless version of the S-procedure [3], the stability condition from Proposition 1 is satisfied if and only 
if there exists $\varepsilon \geq 0$ such that

$\left(\begin{array}{c}\varphi_{\tau}(\sigma, x) \\ x\end{array}\right)^{T} \Omega\left(\begin{array}{c}\varphi_{\tau}(\sigma, x) \\ x\end{array}\right) \leq 0$, with $\Omega$ given in $(6)$. One can finally derive Lemma 3 stability conditions after expressing the evolution of the system state: $\varphi_{\tau}(\sigma, x)=$ $\left(I+\int_{0}^{\sigma} \mathrm{e}^{s A} d s(A-B K)\right) x=\Lambda(\sigma) x$.

Proof of Theorem 4: Let $x$ be in $\mathbb{R}^{n}$. There exists a region $\mathcal{R}_{s}$ as in (8) such that $x \in \mathcal{R}_{s}$ and $\tau(x)=\tau_{s}$. Using the lossless version of the $\mathrm{S}$-procedure, one can see that for any $\kappa \in \mathcal{K}_{s}$ the condition $x^{T} \Phi_{\kappa, s} x \leq 0, x \in \mathcal{R}_{s}$ is satisfied if and only if there exists a scalar $\varepsilon_{\kappa, s} \geq 0$ such that $\Phi_{\kappa, s}+\varepsilon_{\kappa, s} Q_{s} \preceq 0$. Therefore, if the condition $\Phi_{\kappa, s}+$ $\varepsilon_{\kappa, s} Q_{s} \preceq 0$ is satisfied for all $s \in\{1, \cdots, q\}$ and $\kappa \in \mathcal{K}_{s}$, then for all $x \in \mathbb{R}^{n}$, for all $\sigma \in[0, \tau(x)], x^{T} \Phi(\sigma) x \leq$ 0 , according to $(9)$, and the stability conditions from Lemma 3 are satisfied.

Proof of Corollary 5: This comes naturally from Theorem 4 and Proposition 2 when working with a single region: $\mathbb{R}^{n}$ itself.

\subsection{Two methods to build the conic covering}

\subsubsection{Using the spherical coordinates of the state, isotropic covering}

The first conic covering we propose uses the generalized spherical coordinates of the state $x$ in $\mathbb{R}^{n}$ : $\left(r, \theta_{1}, \cdots, \theta_{n-1}\right)$, provided by the relations

$$
\begin{aligned}
r & =\|x\|, \\
x_{1} & =r \cos \theta_{1}, \\
x_{2} & =r \sin \theta_{1} \cos \theta_{2}, \\
& \vdots \\
x_{n-1} & =r \sin \theta_{1} \ldots \sin \theta_{n-2} \cos \theta_{n-1}, \\
x_{n} & =r \sin \theta_{1} \ldots \sin \theta_{n-2} \sin \theta_{n-1},
\end{aligned}
$$

with $\theta_{1}, \theta_{2}, \ldots, \theta_{n-2} \in[0, \pi]$, and $\theta_{n-1} \in[-\pi, \pi]$. Each region $\mathcal{R}_{s}$ is then associated to some range of the $(n-1)$ angular coordinates $\theta_{i}:\left(x \in \mathcal{R}_{s}\right) \Leftrightarrow$ $\left(\forall i \in\{1, \cdots, n-1\}, \theta_{i} \in\left[\theta_{i, s}^{-}, \theta_{i, s}^{+}\right]\right)$. An illustration of such conic regions in $\mathbb{R}^{2}$ is shown in Figure 5 .

Then, in order to build the matrices $Q_{s}$ defining these regions $\mathcal{R}_{s}$ (8), one can use some geometric arguments: if $x \in \mathcal{R}_{s}$, then for $\theta_{i, s}^{-+} \in\left[0, \frac{\pi}{2}\right)$,

$\forall i \in\{1, \cdots, n-2\},\left\{\begin{array}{l}x_{i}^{2} \tan ^{2} \theta_{i, s}^{-} \leq x_{i+1}^{2}+\cdots+x_{n}^{2} \\ x_{i}^{2} \tan ^{2} \theta_{i, s}^{+} \geq x_{i+1}^{2}+\cdots+x_{n}^{2}\end{array}\right.$,

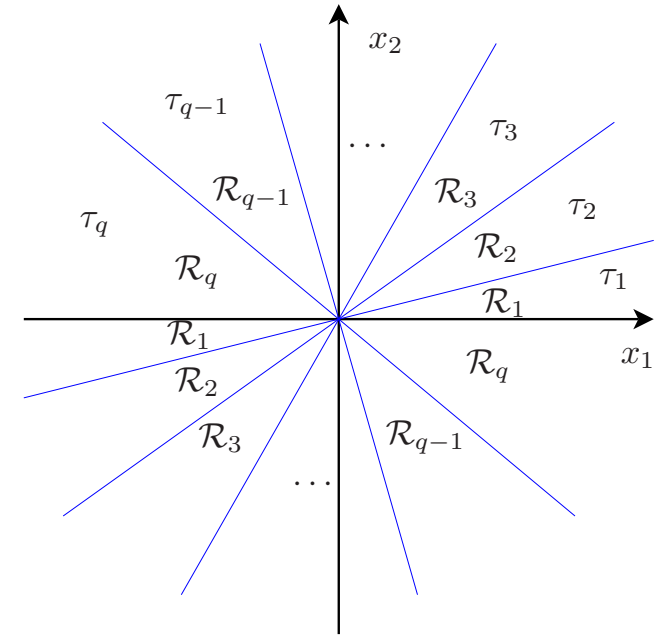

Fig. 5. Covering the state space of dimension 2 with $q$ conic regions $\mathcal{R}_{s}$.

and

$$
\left\{\begin{array}{l}
x_{n-1} \geq \tan \theta_{n-1, s}^{-} x_{n} \\
x_{n-1} \leq \tan \theta_{n-1, s}^{+} x_{n}
\end{array} .\right.
$$

Similar conditions can be obtained for $\theta_{i, s}^{-+} \in\left(\frac{\pi}{2}, \pi\right]$. The design of the conic forms $Q_{s}$ from (11) and (12) is then trivial.

Note that with this covering, the state position is characterized by its only $n-1$ angular coordinates $\theta_{1}, \cdots, \theta_{n-1}$. Thus, situating $x \in \mathbb{R}^{n}$ in this conic covering is easy, which is important since it has to be done in real-time. The computational complexity to calculate the angular coordinates and find the right region is linear in the system's dimension $(O(n))$, and does not depend on the number of regions. More precisely, one can show that $9 n-7$ elementary operations are required (additions, multiplications and divisions), added to 1 square-root, $n-1$ arccosine, and $n-2$ sine. Also, note that the smaller the ranges $\left[\theta_{i, s}^{-}, \theta_{i, s}^{+}\right]$of each conic region, the closer the obtained state-dependent sampling function will be from the optimal sampling function.

A drawback of this covering technique is that the number of regions to be considered exponentially increases with the dimension $n$ of the system. If one divides each angular coordinate range in $m$ equal sectors (what we call "isotropic covering"), this provides a precision of $\frac{\pi}{m} \mathrm{rad}$ for each angle and one needs $m^{n-1}$ conic regions. This means that a tradeoff between the offline computational complexity and the accuracy of the approximation has to be achieved. Furthermore, there is a link between the conservatism of the proposed solution and the accuracy of approximation. 


\subsubsection{Using the discrete-time model behaviour}

A second covering technique involves the dynamics of the discrete-time system. Assume that the conditions from Corollary 5 are satisfied for a given $\tau^{*}=\tau_{\text {sub }}^{*}$. Then, there exists a matrix $P=P^{T} \succ 0$ such that

$$
x^{T}\left(\Lambda^{T}\left(\tau^{*}\right) P \Lambda\left(\tau^{*}\right)-\mathrm{e}^{-2 \beta \tau^{*}} P\right) x \leq 0
$$

is satisfied for all $x \in \mathbb{R}^{n}$, with $\Lambda$ the transition matrix function defined in (7).

The conic regions will be obtained by using the regions described by (13) for values of $\tau$ larger than $\tau^{*}$. For a given scalar $\bar{\sigma}>\tau^{*}$, consider the following set of sampling times $T_{s}=\tau^{*}+(s-1) \frac{\bar{\sigma}-\tau^{*}}{q-1}, s \in\{1, \cdots, q\}$ $\left(\tau^{*} \leq T_{s} \leq \bar{\sigma}\right)$, and design the conic regions as:

$$
\mathcal{R}_{s}=\left\{x \in \mathbb{R}^{n}, x^{T}\left(\Lambda^{T}\left(T_{s}\right) P \Lambda\left(T_{s}\right)-\mathrm{e}^{-2 \beta T_{s}} P\right) x \leq 0\right\} .
$$

Such regions ensure that the function $V(x)=x^{T} P x$ is decreasing at sampling times along the solutions of the discrete-time model

$$
x_{k+1}=\Lambda\left(\tau\left(x_{k}\right)\right) x_{k}, t_{k+1}=t_{k}+\tau\left(x_{k}\right),
$$

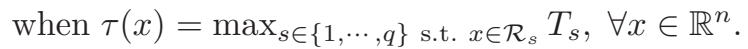

Using Theorem 4 allows us to guarantee the decay of the Lyapunov-Razumikhin function such as in Proposition 1 for the solution of the continuous-time model $\mathcal{S}$. Note that the case $s=1$ corresponds to $\mathcal{R}_{1}=\mathbb{R}^{n}$.

In this construction, the division is achieved on the timevariable $T_{s}$ rather than on angular coordinates. The advantage is that the number of regions does not depend on the dimension of the system and is proportional to the numerical precision, whereas in the previous covering construction, it was an exponential function. The drawback is that more online computation is needed for situating the sampled state in its corresponding conic region: the inequalities $x^{T}\left(\Lambda^{T}\left(T_{s}\right) P \Lambda\left(T_{s}\right)-\mathrm{e}^{-2 \beta T_{s}} P\right) x=$ $x^{T}\left(-Q_{s}\right) x \leq 0$ have to be checked. Thus, with this second construction, the tradeoff moves to offline/online computational effort. At each sampling instant, the number of additions required to find the region is at most $(q-1)(n-1)(n+1)$, and the number of multiplications is at most $(q-1) n(n+1)$. The computational complexity is in $O\left(q n^{2}\right)$.

\subsection{A possible construction of the convex polytope}

Lemma 6 Consider a vector $x \in \mathbb{R}^{n}$, a scalar $\bar{\sigma}>0$, integers $N \geq 0$ and $l \geq 1$, parameters $P=P^{T} \succ 0 \in$ $\mathcal{M}_{n}(\mathbb{R}), \alpha>1,0<\beta \leq \frac{\ln (\alpha)}{2 \bar{\sigma}}$, and $\varepsilon \geq 0$, and a sampling interval $\tau_{s}>0$. If the condition $x^{T} \Phi_{(i, j), s} x \leq 0$ is satisfied for all $(i, j) \in \mathcal{K}_{s}=\left(\{0, \cdots, N\} \times\left\{0, \cdots,\left\lfloor\frac{\tau_{s} l}{\bar{\sigma}}\right\rfloor\right\}\right)$, then for all $\sigma \in\left[0, \tau_{s}\right], x^{T} \Phi(\sigma) x \leq 0$, with $\Phi$ defined in (5) and

$$
\begin{gathered}
\Phi_{(i, j), s}=\hat{\Phi}_{(i, j), s}+\nu I \\
\left\{\begin{array}{l}
\hat{\Phi}_{(i, j), s}=\left(\sum_{k=0}^{i} L_{k, j}\left(\frac{\bar{\sigma}}{l}\right)^{k}\right) \text { if } j<\left\lfloor\frac{\tau_{s} l}{\bar{\sigma}}\right\rfloor \\
\hat{\Phi}_{(i, j), s}=\left(\sum_{k=0}^{i} L_{k, j}\left(\tau_{s}-\frac{j \bar{\sigma}}{l}\right)^{k}\right) \text { otherwise }
\end{array}\right. \\
\left\{\begin{array}{c}
L_{0, j}=\Pi_{3, j}^{T} \Pi_{1} \Pi_{3, j}-\varepsilon P-\Pi_{3, j}^{T} \Pi_{2}-\Pi_{2}^{T} \Pi_{3, j} \\
L_{1, j}=\Pi_{4, j}^{T}\left(\Pi_{1} \Pi_{3, j}-\Pi_{2}\right)+\left(\Pi_{3, j}^{T} \Pi_{1}^{T}-\Pi_{2}^{T}\right) \Pi_{4, j} \\
L_{k \geq 2, j}=\Pi_{4, j}^{T} \frac{\left(A^{k-1}\right)^{T}}{k !}\left(\Pi_{1} \Pi_{3, j}-\Pi_{2}\right) \\
\quad+\left(\Pi_{3, j}^{T} \Pi_{1}^{T}-\Pi_{2}^{T}\right) \frac{A^{k-1}}{k !} \Pi_{4, j} \\
\quad+\Pi_{4, j}^{T}\left(\sum_{i=1}^{k-1} \frac{\left(A^{i-1}\right)^{T}}{i !} \Pi_{1} \frac{A^{k-i-1}}{(k-i) !}\right) \Pi_{4, j} \\
\left\{\begin{array}{c}
\Pi_{1}=A^{T} P+P A+\varepsilon \alpha P+2 \beta P, \Pi_{2}=P B K \\
\Pi_{3, j}=I+M_{j}(A-B K), \Pi_{4, j}=N_{j}(A-B K)
\end{array}\right. \\
M_{j}=\int_{0}^{j \frac{\bar{\sigma}}{l}} e^{A s} d s, N_{j}=A M_{j}+I
\end{array}\right.
\end{gathered}
$$

and

$$
\nu \geq \max _{\substack{\sigma^{\prime} \in[0, \bar{\sigma}], r \in\{0, \cdots, l-1\}}} \lambda_{\max }\left(\Phi\left(\sigma^{\prime}+r \frac{\bar{\sigma}}{l}\right)-\bar{\Phi}_{N, r}\left(\sigma^{\prime}\right)\right)
$$

with the function $\bar{\Phi}_{N, r}$ defined in (23).

\section{Proof:}

(1) First, we divide the time interval $[0, \bar{\sigma}]$ into $l$ subdivisions and take a time $\sigma \leq \tau_{s}$ into one of these subdivisions. The aim of this step is to make preparations to compute a precise estimation of $\Phi($.$) by$ building $l$ small convex embeddings around it instead of building one big one.

(2) Then, we compute a polynomial approximation of $\Phi($.$) for the chosen time interval subdivision.$

(3) Afterwards, we bound the error term from this polynomial approximation with a constant term.

(4) Finally, we build a convex polytope around the polynomial approximation and the error term bound, using the method proposed in [10], Lemma 1 , to obtain the desired finite number of conditions.

Step (1): Let us divide the time interval $[0, \bar{\sigma}]$ into $l$ subdivisions $\left[j \frac{\bar{\sigma}}{l},(j+1) \frac{\bar{\sigma}}{l}\right]$, with $j \in\{0, \cdots, l-1\}$. Let $\sigma \in\left[0, \tau_{s}\right]$. There exists $j \in\left\{0, \cdots,\left\lfloor\frac{\tau_{s} l}{\bar{\sigma}}\right\rfloor\right\}$ such that $j \frac{\bar{\sigma}}{l} \leq \sigma \leq(j+1) \frac{\bar{\sigma}}{l}$. Then define $\sigma^{\prime}=\sigma-j \frac{\bar{\sigma}}{l}\left(\sigma^{\prime} \in[0, \chi]\right.$, with $\chi=\frac{\bar{\sigma}}{l}$ if $j<\left\lfloor\frac{\tau_{s} l}{\bar{\sigma}}\right\rfloor$, and $\chi=\tau_{s}-\frac{j \bar{\sigma}}{l}$ otherwise).

Step (2): We define $\Pi_{1}=A^{T} P+P A+\varepsilon \alpha P+2 \beta P$ and $\Pi_{2}=P B K$. From equations (5) and (6), we deduce that

$$
\Phi(\sigma)=\Lambda(\sigma)^{T} \Pi_{1} \Lambda(\sigma)-\Lambda^{T}(\sigma) \Pi_{2}-\Pi_{2}^{T} \Lambda(\sigma)-\varepsilon P .
$$


In order to derive a useful expression of $\Lambda(\sigma)$ (defined in (7)) as a function of $\sigma^{\prime}$, we use the relation $\int_{0}^{a+b} \mathrm{e}^{A s} d s=\int_{0}^{a} \mathrm{e}^{A s} d s+\int_{0}^{b} \mathrm{e}^{A s} d s\left(A \int_{0}^{a} \mathrm{e}^{A s} d s+I\right)$, which is satisfied for any scalars $a$ and $b$, in order to get

$$
\begin{aligned}
\Lambda(\sigma) & =I+\left(M_{j}+\int_{0}^{\sigma^{\prime}} \mathrm{e}^{A s} d s N_{j}\right)(A-B K) \\
& =\Pi_{3, j}+\int_{0}^{\sigma^{\prime}} \mathrm{e}^{A s} d s \Pi_{4, j},
\end{aligned}
$$

with $M_{j}=\int_{0}^{j \frac{\bar{\sigma}}{l}} \mathrm{e}^{A s} d s, N_{j}=A M_{j}+I, \Pi_{3, j}=I+M_{j}(A-$ $B K)$, and $\Pi_{4, j}=N_{j}(A-B K)$. Then, note that

$$
\int_{0}^{\sigma^{\prime}} \mathrm{e}^{A s} d s=\sum_{i=1}^{\infty} \frac{A^{i-1}}{i !} \sigma^{i} .
$$

Combining equations (20), (21) and (22), one gets $\Phi(\sigma)=\sum_{k=0}^{\infty} L_{k, j} \sigma^{\prime k}$, with the $L_{k, j}$ defined in (16). It is then possible to express a polynomial approximation of order $N$ of $\Phi$ on the interval $\left[j \frac{\bar{\sigma}}{l},(j+1) \frac{\bar{\sigma}}{l}\right]$ as

$$
\bar{\Phi}_{N, j}\left(\sigma^{\prime}\right)=\sum_{k=0}^{N} L_{k, j} \sigma^{\prime k}, \sigma^{\prime} \in\left[0, \frac{\bar{\sigma}}{l}\right] .
$$

Step (3): Let us denote the approximation error term $R_{N, j}\left(\sigma^{\prime}\right)=\Phi(\sigma)-\bar{\Phi}_{N, j}\left(\sigma^{\prime}\right)$. If we can compute a bound with a scalar $\nu$ independent of $\sigma^{\prime}$ such that $R_{N, j}\left(\sigma^{\prime}\right) \preceq$ $\nu I$ then the condition $x^{T}\left(\bar{\Phi}_{N, j}\left(\sigma^{\prime}\right)+\nu I\right) x \leq 0$ will imply that $x^{T} \Phi(\sigma) x \leq 0$. Since $R_{N, j}\left(\sigma^{\prime}\right)=\Phi(\sigma)-\bar{\Phi}_{N, j}\left(\sigma^{\prime}\right)$ is symmetric, then if we denote $\lambda_{\sigma^{\prime}}$ the maximal eigenvalue of $R_{N, j}\left(\sigma^{\prime}\right)$, we have $R_{N, j}\left(\sigma^{\prime}\right) \preceq \lambda_{\sigma^{\prime}} I$. As a consequence, $R_{N, j}\left(\sigma^{\prime}\right) \preceq \nu I$ with $\nu$ a constant defined in (19).

Step (4): Since the function $\bar{\Phi}_{N, j}()+.\nu I:[0, \chi] \rightarrow$ $\mathcal{M}_{n}(\mathbb{R})$ is polynomial, we can use the convex polytope envelope given in [10], Lemma 1, to prove that if $x^{T} \Phi_{(i, j), s} x \leq 0$ for all $i \in\{1, \cdots, n\}$, with $\Phi_{(i, j), s}=$ $\left(\sum_{k=0}^{i} L_{k, j} \chi^{k}\right)+\nu I$, then $x^{T}\left(\bar{\Phi}_{N, j}\left(\sigma^{\prime}\right)+\nu I\right) x \leq 0$ and therefore $x^{T} \Phi(\sigma) x \leq 0$.

\section{References}

[1] A. Anta and P. Tabuada. To sample or not to sample: selftriggered control for nonlinear systems. IEEE Transactions on Automatic Control, 55(9):2030-2042, 2010.

[2] F. Blanchini. Set invariance in control. Automatica, 35(11):1747-1767, 1999.

[3] S. Boyd, L. El-Ghaoui, E. Feron, and V. Balakrishnan. Linear matrix inequalities in system and control theory. SIAM, Studies in Applied Mathematics, 15:23-24, 1994.

[4] M.B.G. Cloosterman, L. Hetel, N. van de Wouw, W.P.M.H. Heemels, J. Daafouz, and H. Nijmeijer. Controller synthesis for networked control systems. Automatica, 46(10):15841594,2010
[5] E. Fridman. A refined input delay approach to sampled-data control. Automatica, 46(2):421-427, 2010.

[6] E. Fridman, A. Seuret, and J.-P Richard. Robust sampleddata stabilization of linear systems: An input delay approach. Automatica, 40(8):1441-1446, 2004.

[7] H. Fujioka. Stability analysis of systems with aperiodic sample-and-hold devices. Automatica, 45(3):771-775, 2009.

[8] W.P.M.H Heemels, J.-H. Sandee, and P.P.J. Van Bosch. Analysis of event-driven controllers for linear systems. International Journal of Control, 81(4):571-590, 2008.

[9] W.P.M.H. Heemels, N. van de Wouw, R.H. Gielen, M.C.F. Donkers, L. Hetel, S. Olaru, M. Lazar, J. Daafouz, and S. Niculescu. Comparison of overapproximation methods for stability analysis of networked control systems. In 13th International Conference on Hybrid Systems: Computation and Control, 2010.

[10] L. Hetel, J. Daafouz, and C. Iung. Lmi control design for a class of exponential uncertain systems with application to network controlled switched systems. In American Control Conference 2007, pages 1401-1406, New York City, USA, 2007.

[11] L. Hetel, A. Kruszewski, W. Perruquetti, and J.-P Richard. Discrete and intersample analysis of systems with aperiodic sampling. IEEE Transactions on Automatic Control, 56(7):1696-1701, 2011.

[12] V. Kolmanovskii and A. Myshkis. Applied theory of functional differential equations. Springer, 1992.

[13] J. Lunze and D. Lehmann. A state-feedback approach to event-based control. Automatica, 46(1):211-215, 2010.

[14] M. Mazo-Jr., A. Anta, and P. Tabuada. On self-triggered control for linear systems: guarantees and complexity. In European Control Conference, Budapest, Hungary, 2009.

[15] M. Mazo-Jr., A. Anta, and P. Tabuada. An iss selftriggered implementation of linear controllers. Automatica, 46(8):1310-1314, 2010.

[16] P. Naghshtabrizi, J.-P. Hespanha, and A.-R. Teel. Exponential stability of impulsive systems with application to uncertain sampled-data systems. Systems and Control Letters, 57(5):378-385, 2008.

[17] A. Seuret. Stability analysis for sampled-data systems with a time-varying period. In Conference on Decision and Control 2009, Shanghai, China, 2009.

[18] P. Tabuada. Event-triggered real-time scheduling of stabilizing control tasks. IEEE Transactions on Automatic Control, 52(9):1680-1685, 2007.

[19] X. Wang and M.D. Lemmon. Self-triggering under stateindependent disturbances. IEEE Transactions on Automatic Control, 55(6):1494-1500, 2010.

[20] W. Zhang, M.S. Branicky, and S.M. Phillips. Stability of networked control systems. IEEE Control Systems Magazine, 21(1):84-99, 2001. 